\title{
Prostate cancer research in India: A scientometric analysis of publications output during 2004-13
}

\author{
Brij Mohan Gupta ${ }^{1}$, Ritu Gupta ${ }^{2}$ \\ ${ }^{1}$ National Institute of Science, Technology \& Development Studies (NISTADS), New Delhi 11012, India \\ ${ }^{2}$ Shri Venkateshwar University, Meerut- 250 002, India
}

Received November 16, 2014; Revised January 16, 2015; Accepted January 20, 2015; Published Online January 22, 2015

\section{Review Article}

\begin{abstract}
This review article examines 1,368 publications on prostate cancer in India, as covered in Scopus database during 2004-13, experiencing an annual average growth rate of $18.77 \%$ and citation impact of 5.23 . The world prostate cancer output (89,994 publications) came from several countries, of which the top 15 (United States, United Kingdom, Germany, Canada, Italy, Japan, and China) accounts for $94.80 \%$ share of the global output during 2004-13. India's global publication share was $1.52 \%$ and hold $14^{\text {th }}$ rank in global publication output during 2004-13. The Indian prostate cancer output came from several organizations and authors, of which the top 20 and 19 contributed $41.81 \%$ and $24.05 \%$ share, respectively, during 2004-13. India's international collaborative share in prostate cancer was $23.39 \%$, which decreased from $24.42 \%$ to $22.98 \%$ from 2004-08 to 2009-13. Medicine accounted for the largest share $(59.50 \%)$ of output in prostate cancer followed by biochemistry, genetics and molecular biology (40.13\%), pharmacology, toxicology \& pharmaceutics (27.63\%), chemistry (8.55\%), agricultural and biological sciences (4.31\% share), and immunology and microbiology (2.70\% share) during 2004-13. Diagnosis, screening, chemotherapy, radiotherapy, pathology and prognosis together account for $60.24 \%$ publications share among treatments methods used in Indian prostate cancer research during 2004-13. Only Delhi, Maharashtra, Uttar Pradesh and Tamil Nadu together contributed 57.82\% share in Indian publications output in prostate cancer during 2004-13. The authors stressed the need for developing national policy for prostate cancer which should take care of screening for detection and diagnosis, management and treatment options of the prostate cancer patients in India.
\end{abstract}

Keywords: Prostate Cancer; Publications; Scientometrics

\section{Introduction}

The prostate gland is part of a man's reproductive and urinary systems. It is oval shaped with a rounded tip and its size varies (with age) from the size of a walnut to a small apple. The prostate surrounds the base (or neck) of the bladder. It has 2 lobes that surround the urethra. The urethra carries urine from the bladder, through the prostate, and out the penis. The prostate gland is covered in a layer of connective tissue called the prostatic capsule and is made up of different types of cells: (i) gland cells that produce the fluid portion of semen, (ii) muscle cells that control urine flow and ejaculation and (iii) fibrous cells that provide the supportive structure of the gland. Around the prostate, there are structures: (i) seminal vesicles - these glands produce semen and are found on both sides of the prostate, (ii) vas deferens - these tubes carry sperm from the testicles to the seminal vesicles, (iii) nerve bundles - these nerves control bladder and erectile function and are found on both sides of the prostate and (iv) muscles -these muscles control urination. ${ }^{1}$
The prostate gland is divided into three zones: (i) peripheral zone- it is the largest part of prostate and closest to the rectum. This zone accounts for approximately $75 \%$ the prostate tumors (approximately 75\%); (ii) transition zone - a middle area of the prostate (about $20 \%$ of the prostate gland) and surrounds the urethra as it passes through the prostate; and (iii) central zone- The area in front of the transition zone of the prostate and is farthest from the rectum. The main function of the prostate is to produce the fluid portion of semen. A thin fluid (made continuously) rich in proteins and minerals that maintain and nourish sperm is produced by the gland cells within the prostate. When a man is sexually aroused, the prostate produces larger amounts of this fluid. It then mixes with sperm and is ejaculated as semen. However, its excess production passes from the body to the urine. The prostate also plays a part in controlling the flow of urine. The urethra runs from the bladder, through the prostate, and out through the penis. The muscle fibers of the prostate are wrapped around the urethra and are under involuntary 
nervous system control. These fibers contract to slow and stop the flow of urine. ${ }^{1}$ Prostate cancer starts in the cells of the prostate several types of cells are found in the prostate, but almost all prostate cancers develop from the gland cells. Gland cells make the prostate fluid that is added to the semen. The cancer that starts in gland cells is called isadenocarcinoma. Other rare types of cancer can also start in the prostate gland, including sarcomas, small cell carcinomas, and transitional cell carcinomas. ${ }^{2}$

Prostate cancer is the second most common cancer (with $15 \%$ of all cancer cases) in men, with an estimated 1.1 million cases (developed region -759,000 cases, developing regions - 353,000, India-19,000) is diagnosed in 2012. The prostate cancer incidence varies across different geographical regions. It is very high in developed regions such as Australia/New Zealand and North America (Age Standardized Rates (ASR) 111.6 and 97.2 per 100,000 respectively). It is high in terms of Age Standarized Rates in few developed regions such as Caribbean (79.8), South Africa (61.8) and South America (60.1) and low in Asian region with estimated rates of 10.5 and 4.5 in Eastern and South Central Asia. With an estimated 307,000 deaths (developed regions-142,000, developing regions-165,000 and India-12,000) in 2012, prostate cancer is the fifth leading cause of death from cancer in men (6.6\% of the total men deaths). Mortality rates in terms of Age Standarized Rates are observed generally high in predominantly black population (Caribbean, 20 per 100,000), Sub-Saharan Africa (ASRs 19-24 per 100,000), very low in Asia (2.9 per 100,000 in South Central Asia) and intermediate in the Americas and Oceania. ${ }^{3}$

In the past, there are no specific studies on the scientometric analysis of prostate cancer research both at global and national level. However, only few studies have been undertaken in the past on the contribution, citation impact, and evaluation of Indian cancer literature. Among such quantitative studies, Patra and Bhattacharya ${ }^{4}$ had analyzed World and Indian oncology research output during 1987-2003, using PubMed database. It studied research output and global contribution of different countries in world output. It mainly studied India's cancer literature growth trends and explored the application of Bradford's Law of Scattering for identifying core journals and Lotka's Law for author productivity in Indian literature. It also identified the most active Indian institutions involved in cancer research. Lewison and Roe ${ }^{5}$ undertook a comprehensive study on Indian cancer research output during 1990-2010. It focused on Indian cancer literature, its growth, research impact, international collaborative publications share, publication output by geographical areas and, type of research, publication output by cancer site or manifestation and sources of funding for Indian research. The authors observed that distribution of Indian research by cancer site correlates positively with the relative disease burden. Basu, Roe and Lewison ${ }^{6}$ studied the Canada and USA settled (NRIs) Indian origin scholars in the field of cancer during three periods: 1988-90, 1998-2000 and 2008-10. Several authors have examined Indian research publications output in bone marrow research, oral cancer ${ }^{8}$ and cervical cancer ${ }^{9}$ and lymphoma cancer ${ }^{10}$. These publications have examined the literature growth, citation impact and global share, the contribution by sub-fields, type of research, cancer site, treatment methods and population age groups, contribution by type of Indian organization and geographical distribution, contribution and citation impact of top organizations and authors and pattern of communication. Keyan et al. 11 examined the articles in 39 issues of Indian Journal of Cancer during 2003-12. It studied the literature growth, author productivity, authorship pattern, average length of articles and country collaboration of cancer research in India.

The main objectives of this study are to study the performance of Indian research in prostate cancer during 2004-13, based on publications output, as indexed in Scopus database. In particular, the study focuses on the following objectives to study

- Growth of world and Indian research output by type of publication;

- Citation pattern of the Indian research output;

- Contribution, global publication share and citation impact of top 15 most productive countries;

- International collaboration share of publication of top 15 most productive countries and the extent of inter-country collaborative linkages among them and to identify the leading collaborative partners and their share in India's output;

- Distribution of Indian research output by broad subject areas and study their growth and decline;

- Indian prostate cancer output by treatment methods and their distribution by geographical areas;

- Publication productivity and citation impact of most productive twenty Indian institutions and nineteen authors; and

- $\quad$ Leading media of communication

\section{Literature search}

The study retrieved and downloaded the publication data of the world and of 15 most productive countries in prostate cancer from the Scopus database (http://www.scopus.com) for 10 years during 2004-13. The keyword "prostate cancer" and "cancer or neoplasm or carcinoma" were used in "title, abstract and keyword" tag and restricting it to the period 2004-13 in "date range tag" was used for searching the global publication data and this become the main search string. When the main search string with restricted to 15 most productive countries in "country tag", as shown below, the publication data on productive countries were obtained. When the main search string is further restricted to "subject area tag", "country tag", "source title tag", "journal title name" and "affiliation tag", we got information on distribution of publications by subject, collaborating countries, organiza- 
tion-wise and journal-wise, etc. For citation data, the three years, two years, one year citation window was used for publications during 2004-11, 2012 and 2013. In addition, citations of publications were also collected from date of publications till the end of July 2014 for sections 4.1 and 4.10.

\section{Literature search results}

The world and India has published 89994 and 1368 publications in prostate cancer during 2004-13, which have increased from 6402 and 55 publications in 2004 to 11739 and 239 publications in 2014, registering an annual average growth rates of $7.87 \%$ and $18.77 \%$. The cumulative growth of world and Indian publication output in prostate cancer research has increased from 37482 and 389 publications during 2004-08 to 52512 and 979 publications during 2009-13, witnessing a growth rate of $40.10 \%$ and $151.67 \%$. India's global publications share in prostate cancer was $1.52 \%$ during 2004-13, which increased from $1.04 \%$ during 2004-08 to $1.86 \%$ during $2009-13$. The average citation per publication registered by Indian publications in prostate cancer was 5.23 during 2004-13, which had increased from 4.91 during 2004-08 to 5.35 during 2009-13. Of the total Indian publications in prostate cancer during 2004-13, articles constituted $67.03 \%$ share, reviews $(21.35 \%)$, letters $(3.07 \%)$, conference publications $(2.70 \%)$, notes $(2.12 \%)$, editorial (1.75\%), nook chapters $(0.80 \%)$, short surveys $(0.80 \%)$ and articles in press (0.07\%) Table 1 .

TABLE 1: World and Indian literature in prostate cancer: Growth, impact \& international collaboration, 2004-13.

\begin{tabular}{ccccccc}
\hline \hline Publication & World & \multicolumn{5}{c}{ India } \\
\cline { 5 - 8 } Year/s & TP & TP & TC & ACPP & ICP & \%ICP \\
\hline 2004 & 6402 & 55 & 246 & 4.47 & 10 & 18.18 \\
2005 & 7013 & 59 & 192 & 3.25 & 10 & 16.95 \\
2006 & 7350 & 77 & 341 & 4.43 & 18 & 23.38 \\
2007 & 8144 & 85 & 471 & 5.54 & 19 & 22.35 \\
2008 & 8573 & 113 & 661 & 5.85 & 38 & 33.63 \\
2009 & 9234 & 127 & 459 & 3.61 & 27 & 21.26 \\
2010 & 9830 & 147 & 778 & 5.29 & 30 & 20.41 \\
2011 & 10390 & 227 & 1700 & 7.49 & 64 & 28.19 \\
2012 & 11319 & 239 & 1935 & 8.10 & 56 & 23.43 \\
2013 & 11739 & 239 & 365 & 1.53 & 48 & 20.08 \\
$2004-08$ & 37482 & 389 & 1911 & 4.91 & 95 & 24.42 \\
$2009-13$ & 52512 & 979 & 5237 & 5.35 & 225 & 22.98 \\
$2004-13$ & 89994 & 1368 & 7148 & 5.23 & 320 & 23.39 \\
\hline TP = Total publications; TC = Total citations; ACPP = Average \\
citations per publication; ICP = International collaborative \\
\multicolumn{7}{c}{ publications } \\
\hline \hline
\end{tabular}

\section{Citation pattern of research output}

Citations of 1368 Indian publications during 2004-13 in prostate cancer were examined since the year of publication till June July 2014. During this period, 14428 citations were received by 1368 publications, leading to the average citation per publication of 10.55 . Only $28.65 \%$ of the total publications did not get any citations (zero citation) and rest of the $71.35 \%$ publications were cited one or more times. Of the total cited publications, 3.29\% publications (receiving more than 50 citations) contributed $34.27 \%$ citations share, $4.02 \%$ publications (received citations from 31 to 50) contributed $14.83 \%$ citations share, $17.91 \%$ publications (received citations from 11 to 30 ) contributed $33.28 \%$ citation share and the rest $46.13 \%$ publications (receiving citations from 1 to 20) contributed $17.63 \%$ citations share (Table 2).

TABLE 2: Citations received by Indian publications in prostate cancer, 2004-13.

\begin{tabular}{ccccc}
\hline \hline $\begin{array}{l}\text { No. of } \\
\text { Citations }\end{array}$ & $\begin{array}{c}\text { No. of } \\
\text { Publications }\end{array}$ & $\begin{array}{c}\text { Total } \\
\text { Citations }^{*}\end{array}$ & $\begin{array}{c}\% \\
\text { Publications }\end{array}$ & $\begin{array}{c}\% \\
\text { Citations }\end{array}$ \\
\hline 0 & 392 & 0 & 28.65 & 0.00 \\
$1-10$ & 631 & 2543 & 46.13 & 17.63 \\
$11-30$ & 245 & 4802 & 17.91 & 33.28 \\
$31-50$ & 55 & 2139 & 4.02 & 14.83 \\
$51-100$ & 32 & 1959 & 2.34 & 13.58 \\
$>100$ & 13 & 2985 & 0.95 & 20.69 \\
Total & 1368 & 14428 & 100.00 & 100.00 \\
\hline \multicolumn{4}{r}{ Citations are from date of publications till July 2014 } \\
\hline \hline
\end{tabular}

Global publication share and citation impact of top 15 most productive countries

The total global research output in prostate cancer originated in 113 countries during 2004-13. Of the 113 countries, 87 countries produced 1 to 50 publications, 10 countries from 51 to 100 publications, 11 countries from 101 to 400 publications, 2 countries from 501 to 700 publications, 2 countries from 1401 to 1800 publications and 1 country between 4801 to 4900 publications during 2004-13.

Table 3 lists the output of top 15 most productive countries that produced $94.80 \%$ of the global output in prostate cancer during 2004-13. The publication share of 15 most productive countries in prostate cancer varied from $1.37 \%$ to $41.56 \%$, with highest publication share $(41.56 \%)$ coming from USA, followed by UK (7.19\% share), Germany (6.64\% share), Canada (5.44\% share), Italy (5.08\% share), Japan $(5.03 \%$ share), China (4.51\% share), France (4.28\% share), Australia, Netherlands, Spain and Sweden (from 2.19 to $2.72 \%$ share), South Korea, India and Switzerland (from 1.37 to $1.97 \%$ share) during 2004-13. The global publication share has increased by $3.23 \%$ in China, followed by Italy (1.01\%), South Korea $(0.95 \%)$, India (0.83\%), Australia (0.78\%), Netherlands (0.49\%), Canada $(0.45 \%)$, Sweden $(0.32 \%)$, France $(0.30 \%)$, Switzerland $(0.21 \%)$ and Spain $(0.08 \%)$, as against decrease by $2.47 \%$ in USA, followed by Japan (1.05\%), Germany $(0.22 \%)$ and UK $(0.12 \%)$ from 2004-08 to 2009-13. Similarly, the global publication rank has increased from $8^{\text {th }}$ to $4^{\text {th }}$ in case of China and Australia (from $11^{\text {th }}$ to $9^{\text {th }}$ ), as against decrease in rank from $4^{\text {th }}$ to $7^{\text {th }}$ in case of Japan and France (from $7^{\text {th }}$ to $8^{\text {th }}$ ) from 2004-08 to 2009-13. The publication rank of other countries has remained the same during this period. 
TABLE 3: Publication output, global publication share and rank of top 15 most productive countries in prostate cancer, 2003-12.

\begin{tabular}{|c|c|c|c|c|c|c|c|c|c|}
\hline \multirow[t]{2}{*}{ Country } & \multicolumn{3}{|c|}{ Number of Publications } & \multicolumn{3}{|c|}{ Global Publication Share } & \multicolumn{3}{|c|}{ Rank } \\
\hline & 2004-08 & 2009-13 & 2009-13 & 2004-08 & 2009-13 & 2009-13 & 2004-08 & 2009-13 & $2004-13$ \\
\hline United States & 16118 & 21287 & 37405 & 43.00 & 40.54 & 41.56 & 1 & 1 & 1 \\
\hline United Kingdom & 2724 & 3751 & 6475 & 7.27 & 7.14 & 7.19 & 2 & 2 & 2 \\
\hline Germany & 2537 & 3441 & 5978 & 6.77 & 6.55 & 6.64 & 3 & 3 & 3 \\
\hline Canada & 1941 & 2958 & 4899 & 5.18 & 5.63 & 5.44 & 5 & 5 & 4 \\
\hline Italy & 1682 & 2888 & 4570 & 4.49 & 5.50 & 5.08 & 6 & 6 & 5 \\
\hline Japan & 2116 & 2412 & 4528 & 5.65 & 4.59 & 5.03 & 4 & 7 & 6 \\
\hline China & 985 & 3077 & 4062 & 2.63 & 5.86 & 4.51 & 8 & 4 & 7 \\
\hline France & 1538 & 2312 & 3850 & 4.10 & 4.40 & 4.28 & 7 & 8 & 8 \\
\hline Australia & 849 & 1600 & 2449 & 2.27 & 3.05 & 2.72 & 11 & 9 & 9 \\
\hline Netherlands & 909 & 1533 & 2442 & 2.43 & 2.92 & 2.71 & 10 & 10 & 10 \\
\hline Spain & 947 & 1367 & 2314 & 2.53 & 2.60 & 2.57 & 9 & 11 & 11 \\
\hline Sweden & 752 & 1221 & 1973 & 2.01 & 2.33 & 2.19 & 12 & 13 & 12 \\
\hline South Korea & 530 & 1241 & 1771 & 1.41 & 2.36 & 1.97 & 13 & 12 & 13 \\
\hline India & 389 & 979 & 1368 & 1.04 & 1.86 & 1.52 & 15 & 14 & 14 \\
\hline Switzerland & 467 & 762 & 1229 & 1.25 & 1.45 & 1.37 & 14 & 15 & 15 \\
\hline World & 37482 & 52512 & 89994 & & & & & & \\
\hline
\end{tabular}

TABLE 4: International collaborative output, share and linkages of top 15 most productive countries during 2004-13.

\begin{tabular}{|c|c|c|c|c|c|}
\hline Country Name & TP & ICP & $\% \mathrm{CP}$ & TCL* & Number of collaborative links with different countries \\
\hline $\begin{array}{l}\text { United States } \\
\text { (US) }\end{array}$ & 37405 & 8787 & 23.49 & 9715 & $\begin{array}{l}\text { UK(1102), GER(1044), CAN(1457), ITL(923), JP(643), CH(1052), FR(616), } \\
\text { AUS(470), NET(499), SPN(407), SWD(668), SKOR(366), IND(216), SWT(252) }\end{array}$ \\
\hline $\begin{array}{l}\text { United Kingdom } \\
\qquad(\mathrm{UK})\end{array}$ & 6475 & 2432 & 37.56 & 4066 & $\begin{array}{l}\text { USA (1102), GER (451), CAN (294), ITL (356),JP (72), CH (68), FR (386), AUS } \\
\text { (215), NET (352), SPN (251), SWD (339), SKOR (23), IND (29), SWT (128) }\end{array}$ \\
\hline Germany (GER) & 5978 & 2424 & 40.55 & 3897 & $\begin{array}{l}\text { USA (1044), UK (451), CAN (326), ITL (410), JP (54), CH (58), FR (332), AUST } \\
\text { (110), NET (327), SPN (187), SWD (310), SKOR (8), IND (24), SWT (256) }\end{array}$ \\
\hline Canada (CAN) & 4899 & 2313 & 47.21 & 3411 & $\begin{array}{l}\text { USA (1457), UK (294), GER (326), ITL (297), JP (82), CH (71), FR (225), AUS } \\
\text { (235), NET (157), SPN (68), SWD (96), SKOR (12), IND (23), SWT (68) }\end{array}$ \\
\hline Italy (ITL) & 4570 & 1802 & 39.43 & 3351 & $\begin{array}{l}\text { USA (923), GER (410), FR (358),UK (356),CAN (297),SPN (297), NET (211), } \\
\text { SWD (188), SWT (169), AUS (61), JP(44), CH (17), IND (11), SKOR (9) }\end{array}$ \\
\hline Japan (JP) & 4528 & 963 & 21.27 & 1252 & $\begin{array}{l}\text { USA(643), CH(110), CAN(82), UK (72), GER (54), ITL(44), FR(42), SKOR(40), } \\
\text { AUS (39), SWD(39), NET(32), SPN(24), IND(16), SWT(15) }\end{array}$ \\
\hline China $(\mathrm{CH})$ & 4062 & 1408 & 34.66 & 1577 & $\begin{array}{l}\text { USA(1052), JP(110), CAN (71), UK(68), GER(58), AUS(52), SWD(38), FR(34), } \\
\operatorname{SKOR(33).~ITL(17),~NET(16),~IND(13),~SPN(9),~SWT(6)~}\end{array}$ \\
\hline France (FR) & 3850 & 1487 & 38.62 & 2839 & $\begin{array}{l}\text { USA(616),UK(386), ITL(358), GER(332), NET(229), CAN(225), SPN(191), } \\
\text { SWD(181), SWT(143), AUS(101),JP(42), CH(34), IND(12), SKOR(9) }\end{array}$ \\
\hline Australia (AUS) & 2449 & 1024 & 41.81 & 1486 & $\begin{array}{l}\text { USA(470), CAN(235), UK(215), GER(110), FR(101), SWD(70), ITL(61), } \\
\text { NET(57), CH(52), JP(39), SPN(35), SWT(16), IND(14), SKOR(11) }\end{array}$ \\
\hline $\begin{array}{l}\text { Netherlands } \\
\text { (NET) }\end{array}$ & 2442 & 1146 & 46.93 & 2312 & $\begin{array}{l}\text { USA(499), UK(352), GER(327), FR(229), ITL(211), SWD(180), CAN(167), } \\
\text { SPN(147), SWT(82), AUS(57), JP(32),CH(16), SKOR(7), IND(6) }\end{array}$ \\
\hline Spain (SPN) & 2314 & 808 & 34.92 & 1810 & $\begin{array}{l}\text { USA(407), ITL (297), UK (251), FR(191), GER(187), NET(147), SWD(120), } \\
\text { CAN (68), SWT(63), AUS (35), JP (24), CH (9), SKOR (7), IND (4) }\end{array}$ \\
\hline Sweden (SWD) & 1973 & 1230 & 62.34 & 2285 & $\begin{array}{l}\text { USA(668), UK(339), GER(310), ITL(188), FR(181), NET(180), SPN(120), } \\
\text { CAN(96), AUS(70), SWT(42), JP(39), CH(38), SKOR (11), IND(3) }\end{array}$ \\
\hline $\begin{array}{l}\text { South Korea } \\
\text { (SKOR) }\end{array}$ & 1771 & 444 & 25.07 & 542 & $\begin{array}{l}\text { USA(366), JP(40), CH(33),UK(23), CAN(12), AUS(11), SWD(11), ITL(9), } \\
\text { FR(9), GER(8), NET(7), SPN(7), IND(4), SWT(2) }\end{array}$ \\
\hline India (IND) & 1368 & 320 & 23.39 & 378 & $\begin{array}{l}\text { USA(216), UK(29), GER(24), CAN(23), JP(16), CH(13), FR(12), ITL(11), } \\
\operatorname{NET(6),~SPN(4),~SKPR(4),~SWD(3),~SWT(3)~}\end{array}$ \\
\hline $\begin{array}{c}\text { Switzerland } \\
(\text { SWT })\end{array}$ & 1229 & 762 & 62.00 & 1245 & $\begin{array}{l}\text { GER(256), USA(252), ITL(169), FR(143), UK(128), , NET(82), CAN(68), } \\
\text { SPN(63), SWD(42), AUS(16), JP(15), CH(6), IND(3), SKOR(2) }\end{array}$ \\
\hline
\end{tabular}




\begin{tabular}{|c|c|c|c|c|c|c|c|}
\hline \multirow[t]{2}{*}{ S.N } & \multirow{2}{*}{$\begin{array}{c}\text { Name of } \\
\text { Collaborative } \\
\text { Country }\end{array}$} & \multicolumn{3}{|c|}{$\begin{array}{l}\text { Number of International } \\
\text { Collaborative Publications }\end{array}$} & \multicolumn{3}{|c|}{$\begin{array}{c}\text { Share of International } \\
\text { Collaborative Publications }\end{array}$} \\
\hline & & 2004-08 & 2009-13 & 2004-13 & 2004-08 & 2009-13 & 2004-13 \\
\hline 1 & USA & 70 & 146 & 216 & 73.68 & 64.89 & 67.50 \\
\hline 2 & U.K. & 8 & 21 & 29 & 8.42 & 9.33 & 9.06 \\
\hline 3 & Germany & 7 & 17 & 24 & 7.37 & 7.56 & 7.50 \\
\hline 4 & Canada & 5 & 18 & 23 & 5.26 & 8.00 & 7.19 \\
\hline 5 & Japan & 5 & 11 & 16 & 5.26 & 4.89 & 5.00 \\
\hline 6 & Saudi Arabia & 2 & 14 & 16 & 2.11 & 6.22 & 5.00 \\
\hline 7 & Australia & 3 & 11 & 14 & 3.16 & 4.89 & 4.38 \\
\hline 8 & China & 2 & 11 & 13 & 2.11 & 4.89 & 4.06 \\
\hline 9 & France & 2 & 10 & 12 & 2.11 & 4.44 & 3.75 \\
\hline \multirow[t]{2}{*}{10} & Italy & 4 & 7 & 11 & 4.21 & 3.11 & 3.44 \\
\hline & & 95 & 225 & 320 & & & \\
\hline
\end{tabular}

Sweden registered the highest share (62.34\%) of international collaborative publications among the top 15 countries in prostate cancer during 2004-13, followed by Switzerland (60.00\%), Canada (47.21\%), Netherlands (46.93\%), Australia (41.81\%), Germany (40.55\%), Italy (39.43\%), France (38.62\%), UK (37.56\%), Spain (34.92\%), China (34.66\%), South Korea (25.70\%), USA (23.49\%), India (23.39\%) and Japan (21.27\%). United States received the highest number (9715) of inter-country international collaboration linkages among the top 15 countries in prostate cancer, followed by UK (4066 linkages), Germany (3897 linkages), Canada (3411 linkages), Italy (3351linkages), France (2839 linkages), Netherlands (2312 linkages), Sweden (2285 linkages), Spain (1810 linkages), China (1577 linkages), Australia (1486 linkages), Japan (1252 linkages), Switzerland (1245 linkages), South Korea (542 linkages) and India (378 linkages) during 2004-13 (Table 4).

The largest number of inter-country collaborative linkages (1457) was between USA-Canada, followed by USA-UK (1102 linkages), USA-China (1052 linkages), USA-Germany (1044 linkages), USA-Italy (923 linkages), USA-Sweden (668 linkages), USA-Japan (643 linkages), USA-France (616 linkages), USA-Netherlands (499 linkages), USA-Australia (470 linkages), UK-Germany (451 linkages), Germany-Italy (410 linkages), USA-Spain (407 linkages), UK-France (386 linkages), USA-South Korea (366 linkages), France-Italy (358 linkages), UK-Italy (356 linkages), Netherlands-UK (352 linkages), UK-Sweden (339 linkages), Germany-France (332 linkages), Germany-Netherlands (327 linkages), Canada-Germany (326 linkages), and so on, during 2004-13 (Table 4). From the above, we can conclude that China, Switzerland South Korea and India have not been preferred partner for international collaboration among the top 15 countries

\section{International collaboration}

The share of international collaborative publications in India's total publication output in prostate cancer research was 23.39\% during 2004-13, which decreased from $24.42 \%$ during
2004-08 to $22.98 \%$ during $2009-13$. India has collaborated with 72 countries in prostate cancer research during 2004-13 and the largest share (67.50\%) was contributed by United States during 2004-13, followed by UK (9.06\%), Germany (7.50\%), Canada (7.19\%), Japan and Saudi Arabia $(5.00 \%$ each), Australia (4.38\%), China (4.06\%), France (3.75\%) and Italy (3.44\%) during 2004-13. The international collaborative publications share increased by $4.11 \%$ in Saudi Arabia, followed by China (2.78\%), Canada (2.74\%), France (2.33\%), Australia (1.73\%). UK (0.91\%) and Germany (0.19), as against decrease by $8.79 \%$ in USA, followed by Italy (1.1\%) and Japan (0.37\%) from 2004-08 to 2009-13 (Table 5).

\section{Subject-wise distribution of research output}

India's prostate cancer research output during 2004-13 has been published in the context of six sub-fields (as reflected in Scopus database classification), with highest publications share $(59.50 \%)$ coming from medicine, followed by biochemistry, genetics \& molecular biology (40.13\%), pharmacology, toxicology \& pharmaceutics (27.63\%), chemistry (8.55\% share), agricultural \& biological sciences $(4.31 \%$ share) and immunology \& microbiology (2.70\% share).

The research activity, as reflected in activity index, has witnessed increase in, pharmacology, toxicology and pharmaceutics, chemistry, agricultural \& biological sciences and immunology \& microbiology in contrast to decrease in medicine and biochemistry, genetics \& molecular biology from 2004-08 to 2009-13. Among these six subjects, the largest citation impact per publication (7.47) was registered by agricultural \& biological sciences, biochemistry, genetics \& molecular biology (6.71), chemistry (6.61), pharmacology, toxicology and pharmaceutics (6.34), medicine (4.83) and immunology \& microbiology (3.89) during 2004-13 (Table 6). 
TABLE 6: Subject-wise break-up of India's publications in prostate cancer, 2003-13.

\begin{tabular}{|c|c|c|c|c|c|c|c|c|c|c|c|}
\hline S.N & Subject Areas & Number & f Publicat & $\mathrm{ns}(\mathrm{TP})^{*}$ & Activit & Index & $\mathrm{TC}$ & ACPP & H-INDEX & ICP(\%ICP) & $\% \mathrm{TP}$ \\
\hline & & 2004-08 & 2004-08 & 2004-08 & 2004-08 & 2009-13 & 2004-13 & 2004-13 & 2004-13 & 2004-13 & 2004-13 \\
\hline 1 & Medicine & 259 & 555 & 814 & 111.90 & 95.27 & 3931 & 4.83 & 38 & $\begin{array}{c}185 \\
(22.73 \%)\end{array}$ & 59.50 \\
\hline 2 & $\begin{array}{l}\text { Biochemistry, } \\
\text { Genetics \& } \\
\text { Molecular } \\
\text { Biology }\end{array}$ & 166 & 383 & 549 & 106.33 & 97.48 & 3683 & 6.71 & 41 & $\begin{array}{c}174 \\
(31.69 \%)\end{array}$ & 40.13 \\
\hline 3 & $\begin{array}{l}\text { Pharmacology, } \\
\text { Toxicology \& } \\
\text { Pharmaceutics }\end{array}$ & 66 & 312 & 378 & 61.40 & 115.34 & 2398 & 6.34 & 32 & $\begin{array}{c}80 \\
(21.16 \%)\end{array}$ & 27.63 \\
\hline 4 & Chemistry & 29 & 88 & 117 & 87.17 & 105.10 & 773 & 6.61 & 22 & $\begin{array}{c}39 \\
(33.33 \%)\end{array}$ & 8.55 \\
\hline 5 & $\begin{array}{l}\text { Agricultural \& } \\
\text { Biological } \\
\text { Sciences }\end{array}$ & 14 & 45 & 59 & 83.45 & 106.58 & 441 & 7.47 & 16 & $\begin{array}{c}24 \\
(40.68 \%)\end{array}$ & 4.31 \\
\hline 6 & $\begin{array}{l}\text { Immunology \& } \\
\text { Microbiology }\end{array}$ & 5 & 32 & 37 & 47.52 & 120.85 & 144 & 3.89 & 9 & $\begin{array}{c}10 \\
(27.03 \%)\end{array}$ & 2.70 \\
\hline & $\begin{array}{l}\text { Total of the } \\
\text { country }\end{array}$ & 389 & 979 & 1368 & 100.00 & 100.00 & & & & & \\
\hline
\end{tabular}

TABLE 7: Distribution of India's prostate cancer publications by treatment methods, 2004-13.

\begin{tabular}{lcccccc}
\hline \hline \multicolumn{1}{c}{ Treatment } & \multicolumn{3}{c}{ Number of Publications } & \multicolumn{3}{c}{ Share of Publications } \\
\multicolumn{1}{c}{ methods } & $\mathbf{2 0 0 4 - 0 8}$ & $\mathbf{2 0 0 9 - 1 3}$ & $\mathbf{2 0 0 4}-13$ & $\mathbf{2 0 0 4 - 0 8}$ & $\mathbf{2 0 0 9 - 1 3}$ & $\mathbf{2 0 0 4}-13$ \\
\hline Diagnosis & 51 & 143 & 194 & 13.11 & 14.61 & 14.18 \\
Screening & 32 & 131 & 163 & 8.23 & 13.38 & 11.92 \\
Chemotherapy & 31 & 107 & 138 & 7.97 & 10.93 & 10.09 \\
Radiotherapy & 29 & 87 & 116 & 7.46 & 8.89 & 8.48 \\
Pathology & 40 & 69 & 109 & 10.28 & 7.05 & 7.97 \\
Prognosis & 21 & 83 & 104 & 5.40 & 8.48 & 7.60 \\
Surgery & 25 & 64 & 89 & 6.43 & 6.54 & 6.51 \\
Genetics & 29 & 39 & 68 & 7.46 & 3.98 & 4.97 \\
Quality of Life & 8 & 37 & 45 & 2.06 & 3.78 & 3.29 \\
Epidemiology & 5 & 13 & 18 & 1.29 & 1.33 & 1.32 \\
Palliative care & 4 & 4 & 8 & 1.03 & 0.41 & 0.58 \\
Total of the country & 389 & 979 & 1368 & & & \\
\hline \hline
\end{tabular}

\section{Prostate cancer publications by treatment methods}

In terms of treatment methods used in prostate cancer during 2004-13, the largest publication share (14.18\%) was registered by diagnosis, followed by screening (11.92\%), chemotherapy $(10.09 \%)$, radiotherapy $(8.48 \%)$, pathology $(7.97 \%)$, prognosis (7.60\%), surgery $(6.51 \%)$, genetics $(4.97 \%)$, epidemiology $(1.32 \%)$ and palliative care $(0.58 \%)$ during $2004-13$. The publication share has increased by $5.15 \%$ (from $8.23 \%$ to $13.38 \%$ ) in screening, followed by $3.08 \%$ ( from $5.40 \%$ to $8.48 \%$ ) in prognosis, $2.96 \%$ (from $7.97 \%$ to $10.93 \%$ ) in chemotherapy, $1.72 \%$ (from $2.06 \%$ to $3.78 \%$ ) in quality of life, $1.50 \%$ (from $13.11 \%$ to $14.61 \%$ ) in diagnosis, $1.43 \%$ (from $7.46 \%$ to $8.89 \%$ ) in radiotherapy, $0.11 \%$ (from $6.43 \%$ to $6.54 \%$ ) in surgery and $0.04 \%$ (from $1.29 \%$ to $1.33 \%$ ) in epidemiology, as against decrease by $3.48 \%$ (from $7.46 \%$ to $3.98 \%$ ) in genetics, $3.23 \%$ (from $10.28 \%$ to $7.05 \%$ ) in pathology and $0.62 \%$ (from $1.03 \%$ to $0.41 \%$ ) in palliative care from 2004-08 to 2009-13 (Table 7).

\section{Geographical distribution of research output}

Among Indian states and union territories contributing to prostate cancer research during 2004-13, the largest publication share $(16.52 \%)$ comes from Delhi, followed by Maharashtra (15.35\%), Uttar Pradesh (15.06\%), Tamil Nadu (10.89\%), Andhra Pradesh (7.38\%), Karnataka (6.94\%), Chandigarh (5.12\%), West Bengal (4.61\%), Punjab (2.41\%), Haryana and Kerala (2.05\% each), Madhya Pradesh (1.75\%), Rajasthan (1.61\%) and Pondicherry (0.51\%).

Together the first four states account for 57.82\% share of Indian publications in prostate cancer during 2004-13. The share of prostate cancer publications have increased by $4.21 \%$ (4.37\% to $8.58 \%$ ) in Andhra Pradesh, $1.53 \%$ (from $0.51 \%$ to $2.04 \%$ ) in Rajasthan, $1.41 \%$ (from $3.60 \%$ to 5.01 ) in West Bengal, $1.08 \%$ (from $6.17 \%$ to $7.25 \%$ ) in Karnataka, $0.85 \%$ (from $10.28 \%$ TO $11.13 \%$ ) in Tamil Nadu and $0.35 \%$ (from $1.80 \%$ to $2.15 \%$ ) in Kerala, as against decrease by $6.73 \%$ (from 
$21.34 \%$ to $14.61 \%$ ) in Delhi, $3.38 \%$ (from $17.48 \%$ to $14.01 \%$ ) in Uttar Pradesh, $1.90 \%$ (16.71\% to $14.81 \%)$ in Maharashtra, $1.09 \%$ ( from $2.83 \%$ to $1.74 \%$ ) in Haryana, $0.72 \%$ (from $1.03 \%$ to $0.31 \%$ ) in Pondicherry, $0.43 \%$ (from $2.06 \%$ to $1.63 \%$ ) in Madhya Pradesh, $0.39 \%$ (from $5.40 \%$ to $5.01 \%$ ) in Chandigarh and $0.22 \%$ (from $2.57 \%$ to $2.35 \%$ ) in Punjab from 2004-08 to 2009-13.(Table 8 )

TABLE 8: Geographical distribution of prostate cancer research publications in India, 2004-13.

\begin{tabular}{|c|c|c|c|c|c|c|}
\hline \multirow{2}{*}{$\begin{array}{l}\text { Name of } \\
\text { Geograph- } \\
\text { ical Area }\end{array}$} & \multicolumn{3}{|c|}{ Number of Publications } & \multicolumn{3}{|c|}{ Share of Publications } \\
\hline & $\begin{array}{c}2004- \\
08 \\
\end{array}$ & $\begin{array}{c}2009- \\
13 \\
\end{array}$ & $\begin{array}{c}2004- \\
13 \\
\end{array}$ & $\begin{array}{c}2004- \\
08 \\
\end{array}$ & $\begin{array}{c}2009- \\
13 \\
\end{array}$ & $\begin{array}{c}2004- \\
13\end{array}$ \\
\hline Delhi & 83 & 143 & 226 & 21.34 & 14.61 & 16.52 \\
\hline $\begin{array}{l}\text { Maha- } \\
\text { rashtra }\end{array}$ & 65 & 145 & 210 & 16.71 & 14.81 & 15.35 \\
\hline $\begin{array}{l}\text { Uttar } \\
\text { Pradesh }\end{array}$ & 68 & 138 & 206 & 17.48 & 14.10 & 15.06 \\
\hline $\begin{array}{l}\text { Tamil } \\
\text { Nadu }\end{array}$ & 40 & 109 & 149 & 10.28 & 11.13 & 10.89 \\
\hline $\begin{array}{l}\text { Andhra } \\
\text { Pradesh }\end{array}$ & 17 & 84 & 101 & 4.37 & 8.58 & 7.38 \\
\hline Karnataka & 24 & 71 & 95 & 6.17 & 7.25 & 6.94 \\
\hline $\begin{array}{l}\text { Chandi- } \\
\text { garh }\end{array}$ & 21 & 49 & 70 & 5.40 & 5.01 & 5.12 \\
\hline West & 14 & 49 & 63 & 3.60 & 5.01 & 4.61 \\
\hline Bengal & & & & & & \\
\hline Punjab & 10 & 23 & 33 & 2.57 & 2.35 & 2.41 \\
\hline Haryana & 11 & 17 & 28 & 2.83 & 1.74 & 2.05 \\
\hline Kerala & 7 & 21 & 28 & 1.80 & 2.15 & 2.05 \\
\hline Madhya & 8 & 16 & 24 & 2.06 & 1.63 & 1.75 \\
\hline Pradesh & & & & & & \\
\hline Rajasthan & 2 & 20 & 22 & 0.51 & 2.04 & 1.61 \\
\hline $\begin{array}{l}\text { Pondi- } \\
\text { cherry }\end{array}$ & 4 & 3 & 7 & 1.03 & 0.31 & 0.51 \\
\hline & 389 & 979 & 1368 & & & \\
\hline
\end{tabular}

\section{Profile of top 20 most productive organizations}

The productivity of 20 most productive Indian organizations in prostate cancer varied from 11 to 90 publications and together contributed $41.81 \%$ (572 publications) share in the cumulative publications output of India in prostate cancer research during 2004-13. The scientometric profile of these 20 Indian organizations is presented in Table 9. Seven organizations have registered higher publications output than the group average of 28.60: AIIMS-New Delhi (90 publications), SGPGIMS-Lucknow (59 publications), TMC-Mumbai (44 publications), PGIMER- Chandigarh (42 publications), IIIM-Srinagar (39 publications), CSMUNIV- Lucknow (35 publications) and IICT-Hyderabad (34 publications). Six organizations have registered more than the average citation per publication (7.31) of 20 organizations: IITR-Lucknow (18.53), SGPGIMS-Lucknow (16.19), AIIMS-New Delhi (11.54), JNU-New Delhi (10.74), NIPER-Mohali (9.33) and CCMB-Hyderabad (8.45) during 2004-13.

Ten organizations have registered more than the average h-index (8.95) of all 20 organizations during 2004-13:
AIIMS-New Delhi (17), SGPGIMS-Lucknow (14), JNU-New Delhi and University of Madras (13 each), IITR-Lucknow (12), JNU-New Delhi, IICT-Hyderabad and IIIM-Srinagar (10 each), CDRI-Lucknow and TMH-Mumbai (9 each) during 2004-13. Eight organizations have achieved more than the average share of international collaborative publications (19.23\%) of all 20 organizations: JNU-New Delhi (63.16\%), AMU-Aligarh (46.15\%), IISc-Bangalore (33.33\%), GNDUNIV-Amritsar (30.77\%), CDRI-Lucknow (29.41\%), BARC-Mumbai (25.00\%), IICT- Hyderabad (23.53\%) and TMH-Mumbai (20.45\%) during 2004-13.

The largest number of national collaborating linkages (15) among the most productive organizations were made by Panjab University (PUNJU), Chandigarh with five organizations (PGIMER-6, AIIMS-5, IIIM-2, GNDUNIV-1 AND NIPER-1), followed by Postgraduate Institute of Medical Education \& Research, Chandigarh with 14 linkages with 3 organizations (AIIMS-6, PUNJU-6 and NIPER-2), All India Institute of Medical Sciences, New Delhi with 13 linkages with 3 organizations (PGIMER-6, PUNJU-5 and JNU-2), CS Maharaj Medical University (CSMUNIV), Lucknow with 11 linkages with 2 organizations (CDRI-8 and SGPGIMS-5), Central Drug Research Institute, Lucknow with 8 linkages with 3 organizations (CSMMUNIV-6, CCMB-1 and IITR-1), Indian Institute of Chemical Technology, Hyderabad with 8 linkages with 3 organizations (CSMMUNIV-6, CCMB-1 and IITR-1), Sanjay Gandhi Postgraduate Institute pf Medical Education \& Research, Lucknow with 7 linkages with 2 organizations (CSMMUNIV-5 and CCMB-2), Tata Memorial Center, Mumbai with 7 linkages with 2 organizations (CSMMUNIV-5 and CCMB-2), Guru Nanak Dev University, Amritsar with 6 linkages with 2 organizations (IIIM-5 and PUNJU-1), Centre for Cellular \& Molecular Biology, Hyderabad with 5 linkages with 3 organizations (IICT-2, SGPGIMS-2 and CDRI-1), National Institute of Pharmaceutical Education \& Research, Mohali with 3 linkages with 2 organizations (PGIMER-2 and PUNJU-1) and Tata Memorial Centre, Mumbai, with 2 linkages with BARC, Mumbai.

\section{Profile of top 19 most productive Indian authors}

The top 19 most productive Indian authors have published 11 to 44 publications each and together contributed $24.05 \%$ (329 publications) share in the cumulative publications output of India in prostate cancer research during 2004-13. The scientometric profile of these 19 Indian authors is presented in Table 10 .

Six authors have registered higher publications per author than the group average (17.32): Mittal RD (44 publications), Saxena AK (25 publications), Gupta NP (22 publications), Aruna Karan (22), Mandhani A (21) and Kumar R (6 publications). Eight authors have registered more than the average citation per publication (6.43) of all 19 authors during 2003-12: Mandal RK (18.60), Singh RP (13.58), Padhye S 
(12.53), Mandal RK (9.00), Arunkumar A (7.33), Vijayababu MR (7.29), Aruna Karan (7.14) and Kamal A (6.92) during 2004-13. Eight authors have registered more than the average h-index (9.32) of all 19 authors during 2003-12: Mittal RD and Aruna Karan with h-index of 14 each, followed by Vijayababu MR and Shukla y (12 each), Singh RP and Arunkumar A (11 each), Mandhani A and Padhye S (10 each). Four authors have achieved more than the average share of international collaborative publications (17.33\%) of all 19 authors: Padhye S (100.00\%), Singh RP (83.33\%), Kamal A (25.00\%) and Hemal AK (25.0\%)

\section{Media of communication}

The 20 most productive journals contributed from 8 to 61 papers and together contributed $24.93 \%$ share (341 papers) to the total journal publication output in prostate cancer during 2004-13. The publication share of these top 15 most productive journals decreased from $27.25 \%$ to $24.00 \%$ from 2004-08 to 2009-13. Of these 20 journals, the highest citation impact per paper of 45.89 by Cancer Research, followed by Molecular \& Cellular Biochemistry (21.11), PLOS One (20.35), BJU International (14.56), European Journal of Medicinal Chemistry (13.76), Bioorganic \& Medicinal Chemistry Letters (12.84), Urology (6.56), Asia Pacific Journal of Cancer Prevention (6.06), etc.

TABLE 9: Scientometric profile of top 15 most productive Indian organizations in prostate cancer, 2004-13.

\begin{tabular}{|c|c|c|c|c|c|c|c|c|}
\hline S.N & Name of Organization & TP & TC & ACPP & ICP & $\% \mathrm{ICP}$ & $\mathrm{HI}$ & $\begin{array}{l}\mathrm{NCL} \\
(\mathrm{NO})\end{array}$ \\
\hline 1 & $\begin{array}{l}\text { All India Institute of Medical Sci- } \\
\text { ences, New Delhi. }\end{array}$ & 90 & 1039 & 11.54 & 16 & 17.78 & 17 & $13(3)$ \\
\hline 2 & $\begin{array}{l}\text { Sanjay Gandhi Postgraduate Insti- } \\
\text { tute of Medical Sciences, Lucknow. }\end{array}$ & 59 & 955 & 16.19 & 4 & 6.78 & 14 & $7(2)$ \\
\hline 3 & Tata Memorial Hospital, Mumbai & 44 & 136 & 3.09 & 9 & 20.45 & 9 & $2(1)$ \\
\hline 4 & $\begin{array}{l}\text { Postgrduate Institute of Medical } \\
\text { Education \& Research, Chandigarh. }\end{array}$ & 42 & 77 & 1.83 & 7 & 16.67 & 8 & $14(3)$ \\
\hline 5 & $\begin{array}{l}\text { Indian Institute of Integrated } \\
\text { Medicine, Srinagar. }\end{array}$ & 39 & 183 & 4.69 & 5 & 12.82 & 10 & \\
\hline 6 & $\begin{array}{l}\text { CS Maharaj Medical University, } \\
\text { Lucknow. }\end{array}$ & 35 & 89 & 2.54 & 5 & 14.29 & 6 & $11(2)$ \\
\hline 7 & $\begin{array}{l}\text { Indian Institute of Chemical } \\
\text { Technology, Hyderabad. }\end{array}$ & 34 & 165 & 4.85 & 8 & 23.53 & 10 & $8(3)$ \\
\hline 8 & University of Madras. & 27 & 160 & 5.93 & 4 & 14.81 & 13 & 0 \\
\hline 9 & Panjab University, Chandigarh. & 27 & 163 & 6.04 & 5 & 18.52 & 10 & $15(5)$ \\
\hline 10 & Christian Medical College, Vellore. & 24 & 11 & 0.46 & 2 & 8.33 & 2 & 0 \\
\hline 11 & $\begin{array}{l}\text { Bhabha Atomic Research Center, } \\
\text { Mumbai. }\end{array}$ & 20 & 88 & 4.40 & 5 & 25.00 & 8 & $2(1)$ \\
\hline 12 & $\begin{array}{l}\text { Jawaharlal Nehru University, New } \\
\text { Delhi. }\end{array}$ & 19 & 204 & 10.74 & 12 & 63.16 & 13 & $2(1)$ \\
\hline 13 & $\begin{array}{l}\text { Central Drugs Research Institute, } \\
\text { Lucknow. }\end{array}$ & 17 & 110 & 6.47 & 5 & 29.41 & 9 & $8(3)$ \\
\hline 14 & $\begin{array}{l}\text { Indian Institute of Toxicological } \\
\text { Research, Lucknow. }\end{array}$ & 15 & 278 & 18.53 & 2 & 13.33 & 12 & \\
\hline 15 & $\begin{array}{l}\text { National Institute of Pharmaceuti- } \\
\text { cal Education \& Research, Mohali. }\end{array}$ & 15 & 140 & 9.33 & 2 & 13.33 & 7 & $3(2)$ \\
\hline 16 & $\begin{array}{l}\text { Indian Institute of Science, Banga- } \\
\text { lore }\end{array}$ & 15 & 80 & 5.33 & 5 & 33.33 & 5 & \\
\hline 17 & $\begin{array}{l}\text { Guru Nanak Dev University, Am- } \\
\text { ritsar }\end{array}$ & 13 & 85 & 6.54 & 4 & 30.77 & 7 & $6(2)$ \\
\hline 18 & $\begin{array}{l}\text { Jawaharlal Nehru Technological } \\
\text { University, Hyderabad }\end{array}$ & 13 & 33 & 2.54 & 2 & 15.38 & 4 & \\
\hline 19 & $\begin{array}{l}\text { Aligarh Muslim University, Ali- } \\
\text { garh }\end{array}$ & 13 & 90 & 6.92 & 6 & 46.15 & 7 & \\
\hline 20 & $\begin{array}{l}\text { Centre for Cellular \& Molecular } \\
\text { Biology, Hyderabad }\end{array}$ & 11 & 93 & 8.45 & 2 & 18.18 & 8 & $5(3)$ \\
\hline & Total of 20 top organizations & 572 & 4179 & 7.31 & 110 & 19.23 & 8.95 & \\
\hline & Total of the country & 1368 & & & & & & \\
\hline & $\begin{array}{l}\text { Share of top } 20 \text { organizations in } \\
\text { India's output }\end{array}$ & 41.81 & & & & & & \\
\hline
\end{tabular}


TABLE 10: Scientometric profile of top 19 Indian authors in prostate cancer, 2004-13.

\begin{tabular}{|c|c|c|c|c|c|c|c|c|}
\hline S.N & Name & Affiliation & TP & TC & ACPP & ICP & \%ICP & $\mathrm{HI}$ \\
\hline 1 & Mittal RD & $\begin{array}{l}\text { Sanjay Gandhi Postgraduate Institute of Medical Sci- } \\
\text { ences, Lucknow }\end{array}$ & 44 & 243 & 5.52 & 3 & 6.82 & 14 \\
\hline 2 & Saxena AK & Indian Institute of Integrated Medicine, Srinagar & 25 & 131 & 5.24 & 4 & 16.00 & 9 \\
\hline 3 & Gupta NP & All India Institute of Medical Sciences, New Delhi & 22 & 92 & 4.18 & 2 & 9.09 & 9 \\
\hline 4 & Karan A & $\begin{array}{l}\text { ALM Postgraduate Institute of Basic Medical Sciences, } \\
\text { University of Madras, Madras }\end{array}$ & 22 & 157 & 7.14 & 3 & 13.64 & 14 \\
\hline 5 & Mandhani A & $\begin{array}{l}\text { Sanjay Gandhi Postgraduate Institute of Medical Sci- } \\
\text { ences, Lucknow }\end{array}$ & 21 & 73 & 3.48 & 2 & 9.52 & 10 \\
\hline 6 & Kumar R & All India Institute of Medical Sciences, New Delhi & 21 & 71 & 3.38 & 2 & 9.52 & 8 \\
\hline 7 & Seth A & All India Institute of Medical Sciences, New Delhi & 16 & 82 & 5.13 & 1 & 6.25 & 8 \\
\hline 8 & Shukla Y & Indian Institute of Toxicological Research, Lucknow & 15 & 279 & 18.60 & 2 & 13.33 & 12 \\
\hline 9 & Mandal RK & $\begin{array}{l}\text { Sanjay Gandhi Postgraduate Institute of Medical Sci- } \\
\text { ences, Lucknow }\end{array}$ & 15 & 135 & 9.00 & 0 & 0.00 & 8 \\
\hline 10 & Padhye S & University of Pune & 15 & 188 & 12.53 & 15 & $\begin{array}{c}100.0 \\
0\end{array}$ & 10 \\
\hline 11 & Vijayababu MR & $\begin{array}{l}\text { ALM Postgraduate Institute of Basic Medical Sciences, } \\
\text { University of Madras, Madras }\end{array}$ & 14 & 102 & 7.29 & 2 & 14.29 & 12 \\
\hline 12 & Goel A & CS Maharaj Medical University, Lucknow & 14 & 22 & 1.57 & 0 & 0.00 & 4 \\
\hline 13 & $\begin{array}{l}\text { Jagannathan } \\
\text { NR }\end{array}$ & All India Institute of Medical Sciences, New Delhi & 14 & 57 & 4.07 & 2 & 14.29 & 8 \\
\hline 14 & Singh RP & Jawaharlal Nehru University New Delhi & 12 & 163 & 13.58 & 10 & 83.33 & 11 \\
\hline 15 & Nayyar R & All India Institute of Medical Sciences, New Delhi & 12 & 39 & 3.25 & 0 & 0.00 & 6 \\
\hline 16 & Kamal A & Indian Institute of Chemical Technology, Hyderabad & 12 & 83 & 6.92 & 3 & 25.00 & 8 \\
\hline 17 & Arunkumar A & $\begin{array}{l}\text { ALM Postgraduate Institute of Basic Medical Sciences, } \\
\text { University of Madras, Madras }\end{array}$ & 12 & 88 & 7.33 & 2 & 16.67 & 11 \\
\hline 18 & Hemal AK & All India Institute of Medical Sciences, New Delhi & 12 & 56 & 4.67 & 3 & 25.00 & 8 \\
\hline 19 & Thulkar S & All India Institute of Medical Sciences, New Delhi & 11 & 56 & 5.09 & 1. & 9.09 & 7 \\
\hline & Total output & & 329 & 2117 & 6.43 & 57 & 17.33 & 9.32 \\
\hline & \multicolumn{2}{|c|}{ Total output of India } & 1368 & & & & & \\
\hline & \multicolumn{2}{|c|}{ Share of 19 authors in India's output } & 24.05 & & & & & \\
\hline
\end{tabular}

In Summary, India has contributed 1368 publications in prostate cancer, which has increased from 55 to 239 publications from the year 2004 to 2013, registering an annual average growth rate of $18.77 \%$. India's publications in prostate cancer have registered citation impact per publication of 5.23 during 2004-13, which has increased from 4.91 during 2004-08 to 5.35 during 2009-13. India is ranked at $14^{\text {th }}$ place in global research output, with global publications share of $1.52 \%$ during 2004-13, increasing from 1.04\% during 2004-08 to $1.86 \%$ during $2009-13$. India's share of international collaborative publications in prostate cancer was $23.39 \%$ during 2004-13, which has decreased from $24.42 \%$ to $22.98 \%$ from 2004-08 to $2009-13$. Only $71.35 \%$ of the total publications received 1 or more citations since their publications till June 2014. Of the total cited publications, 3.29\% publications contributed $34.27 \%$ citations share, $4.02 \%$ publications contributed $14.83 \%$ citations share, $17.91 \%$ publications contributed $33.28 \%$ citation share and the rest $46.13 \%$ publications contributed $17.63 \%$ citations share during 2004-13. In terms of subject-wise contribution, the largest publication share $(59.50 \%)$ comes from medicine, followed by biochemistry, genetics \& molecular biology (40.13\%), pharmacology, toxicology \& pharmaceutics (27.63\%), chemistry (8.55\%), agricultural \& biological sciences (4.31\% share) and immunology \& microbiology (2.70\% share)during 2004-13. The largest citation impact per publication (7.47) was made by agricultural \& biological sciences, biochemistry, genetics \& molecular biology (6.71), chemistry (6.61), pharmacology, toxicology and pharmaceutics (6.34), medicine (4.83) and immunology \& microbiology (3.89) during 2004-13. In terms of treatment methods used in prostate cancer research, the largest publications share $(14.18 \%)$ comes from diagnosis, followed by screening (11.92\%), chemotherapy (10.09\%), radiotherapy $(8.48 \%)$, pathology $(7.97 \%)$, prognosis $(7.60 \%)$, surgery (6.51\%), genetics (4.97\%) during 2004-13. Delhi among Indian states \& union territories, contributed the largest share $(16.52 \%)$ to prostate cancer research, followed by Maharashtra (15.35\%), Uttar Pradesh (15.06\%), Tamil Nadu (10.89\%), Andhra Pradesh (7.38\%), Karnataka (6.94\%), Chandigarh (5.12\%), West Bengal (4.61\%), etc during 2003-12. The 20 most productive Indian organizations contributing to prostate cancer research has contributed 572 publications, accounting for $41.81 \%$ share of the country's publication output. 
TABLE 11: Contribution and citation impact of journals where Indian scholars have contributed during 2004-13.

\begin{tabular}{|c|c|c|c|c|c|c|}
\hline \multirow[t]{2}{*}{ S.N } & \multirow[t]{2}{*}{ Name of the Journal } & \multicolumn{3}{|c|}{ Number of Publications (TP) } & \multirow{2}{*}{$\begin{array}{c}\mathrm{TC}^{*} \\
2004-13\end{array}$} & \multirow{2}{*}{$\begin{array}{c}\text { ACPP } \\
2004-13\end{array}$} \\
\hline & & 2004-08 & 2009-13 & 2004-13 & & \\
\hline 1 & Indian Journal of Urology & 22 & 39 & 61 & 64 & 1.05 \\
\hline 2 & Asia Pacific Journal of Cancer Prevention & 12 & 21 & 33 & 200 & 6.06 \\
\hline 3 & Journal of Cancer Research \& Therapeutics & 5 & 19 & 24 & 92 & 3.83 \\
\hline 4 & Indian Journal of Cancer & 6 & 18 & 24 & 116 & 4.83 \\
\hline 5 & European Journal of Medicinal Chemistry & 2 & 19 & 21 & 289 & 13.76 \\
\hline 6 & Indian Journal of Pathology \& Microbiology & 14 & 5 & 19 & 46 & 2.42 \\
\hline 7 & Bioorganic \& Medicinal Chemistry Letters & 3 & 16 & 19 & 244 & 12.84 \\
\hline 8 & PLOS One & 1 & 16 & 17 & 346 & 20.35 \\
\hline 9 & $\begin{array}{l}\text { International Journal of Pharmaceutical Sciences Review \& } \\
\text { Research }\end{array}$ & 0 & 16 & 16 & 6 & 0.38 \\
\hline 10 & International Journal of Pharmacy \& Pharmaceutical Sciences & 0 & 12 & 12 & 9 & 0.75 \\
\hline 11 & Indian Journal of Clinical Biochemistry & 6 & 6 & 12 & 54 & 4.50 \\
\hline 12 & Journal of International Medical Science Academy & 7 & 4 & 11 & 0 & 0.00 \\
\hline 13 & International Journal of Pharma \& Biosciences & 0 & 10 & 10 & 14 & 1.40 \\
\hline 14 & Molecular \& Cellular Biochemistry & 4 & 5 & 9 & 190 & 21.11 \\
\hline 15 & National Medical Journal of India & 3 & 6 & 9 & 31 & 3.44 \\
\hline 16 & Cancer Research & 8 & 1 & 9 & 413 & 45.89 \\
\hline 17 & Journal of Medical Physics & 2 & 7 & 9 & 23 & 2.56 \\
\hline 18 & BJU International & 6 & 3 & 9 & 131 & 14.56 \\
\hline 19 & Urology & 5 & 4 & 9 & 59 & 6.56 \\
\hline 20 & Indian Journal of Medical Pediatrics \& Oncology & 0 & 8 & 8 & 23 & 2.88 \\
\hline & Total of 20 journals & 106 & 235 & 341 & 2350 & \\
\hline & Total of India in journal output & 389 & 979 & 1368 & & \\
\hline & Share of 20 journals in India's journal output & 27.25 & 24.00 & 24.93 & & \\
\hline & $\begin{array}{r}\mathrm{TP}=\text { Total publications; } \mathrm{TC}=\text { Total citations; } \mathrm{ACl} \\
{ }^{*} \text { Citations of publications are from date o }\end{array}$ & $\begin{array}{l}=\text { Avera } \\
\text { ublicatio }\end{array}$ & $\begin{array}{l}\text { citation p } \\
\text { until July }\end{array}$ & publicati & & \\
\hline
\end{tabular}

The average productivity, average citation impact per publication, h-index and share of international collaborative publications of the 15 most productive Indian organizations were 26.80, 7.31, 8.95 and $19.23 \%$ during 2004-13. The 19 most productive Indian authors contributing to prostate cancer research has contributed 329 publications, accounting for $24.05 \%$ share of the country's publication output. The average productivity, average citation impact per publication, $\mathrm{h}$-index and share of international collaborative publications of the 15 most productive Indian authors were 17.32, 6.43, 9.32 and $17.33 \%$ during 2003-12.

\section{Conclusion}

In order to increase the national output and international collaboration and improve citation impact, there is an urgent need to increase awareness and understanding of the burden of disease associated with prostate cancer, undertake the evaluation of existed prostate cancer related programs and establish a national prostate cancer program at the national level in India. It is also important to develop a systematic screening program for detection and diagnosis of prostate cancer cases to be followed by evolution of suitable management strategy and more effective treatment options for treatment of prostate cancer. There is also need to increase funding support for research and development (R\&D) and develop training programs for concerned persons at different levels.

\section{Conflict of interest}

The authors declare that they have no conflicts of interest. The authors alone are responsible for the content and writing of the paper.

\section{References}

1. Canadian Cancer Society. Prostate cancer. Anatomy and physiology of the prostate. 2014. Available from http://www.cancer.ca/en/cancer-information/cancertype/prostate/anatomy-and-physiology/?region=on

2. American Cancer Society. What is prostate cancer? 2014. Available from

http://www.cancer.org/cancer/prostatecancer/detaile dguide/prostate-cancer-what-is-prostate-cancer

3. WHO \& International Agency for Research on Cancer. GLOBOCAN 2012: Estimated cancer incidence, mortality and prevalence worldwide in 2012. Cancer Fact Sheet. Prostate Cancer. IARC 2014. Available from http://globocan.iarc.fr/Pages/fact_sheets_cancer.aspx

4. Patra SK, Bhattacharya P. Bibliometric study of cancer research in India. DESIDOC Bulletin of Information Technology 2005; 25:11-8.

5. Lewison $G$, Roe P. The evaluation of Indian cancer research, 1990-2010. Scientometrics2012; 93: 167-81 
6. Basu A, Roe P, Lewison G. The Indian diaspora in cancer research: A bibliometric assessment for Canada and the USA. 2014, Available from http://sticonference.org/Proceedings/vol1/Basu_India n_110.pdf

7. Gupta BM, Bala A. Bone marrow research in India: A scientometric study, 2003-12. Journal of Bone Marrow Research 2013; 1: 108.

8. Gupta R, Gupta BM, Ahmed M, Tiwari R. Oral cancer research: A scientometric assessment of Indian publications output during 2003-12. Onc Gas Hep Rep 2014; 3: 14-22.
9. Gupta R, Gupta BM, Ahmed M, Tiwari R. Cervical cancer in India: A scientometric study of publications, 2003-12. Oncology, Onc Gas Hep Rep 2014; 3: 4-13.

10. Gupta R, Gupta, BM. Indian lymphoma research: A scientometric analysis of Indian research output during 2004-13. COLLNET Journal of Scientometrics \& Information Management 2014; 8: 419-436.

11. Santhanakarthikeyan S, Grace M, Jeyshankar R. Research publications to Indian Journal of Cancer: a scientometric analysis. Library Hi Tech News 2014; 31: 21-5. 